Supplementary Information to:

\title{
Ozonolysis of oleic acid aerosol revisited: multiphase chemical kinetics and reaction mechanisms
}

Thomas Berkemeier ${ }^{1, *}$, Ashmi Mishra ${ }^{1}$, Coraline Mattei ${ }^{1}$, Andrew J. Huisman ${ }^{2, \neq}$, Ulrich K. Krieger $^{2}$, Ulrich Pöschl ${ }^{1, *}$

[1] Multiphase Chemistry Department, Max Planck Institute for Chemistry, Hahn-MeitnerWeg 1, Mainz, Germany;

[2] ETH Zurich, Institute for Atmospheric and Climate Science, Universitätstrasse 16, 8092, Zurich, Switzerland;

[‡] now at: Gentex Corporation, Applied Engineering Department, 600 N Centennial St, 49464, Zeeland, Michigan, USA

Correspondence to:

Thomas Berkemeier (t.berkemeier@mpic.de),

Ulrich Pöschl (u.poschl@mpic.de) 


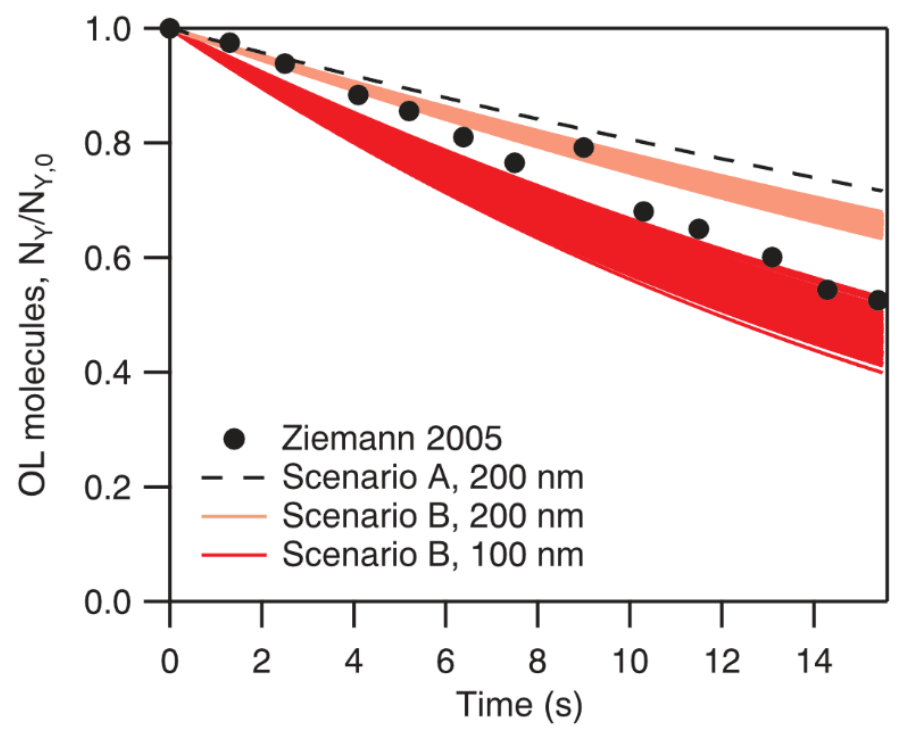

Figure S1: Model sensitivity towards different particle sizes in the EC1 experiment (black markers) in modelling scenario A (dashed line) and modelling scenario B (ensemble of colored lines).
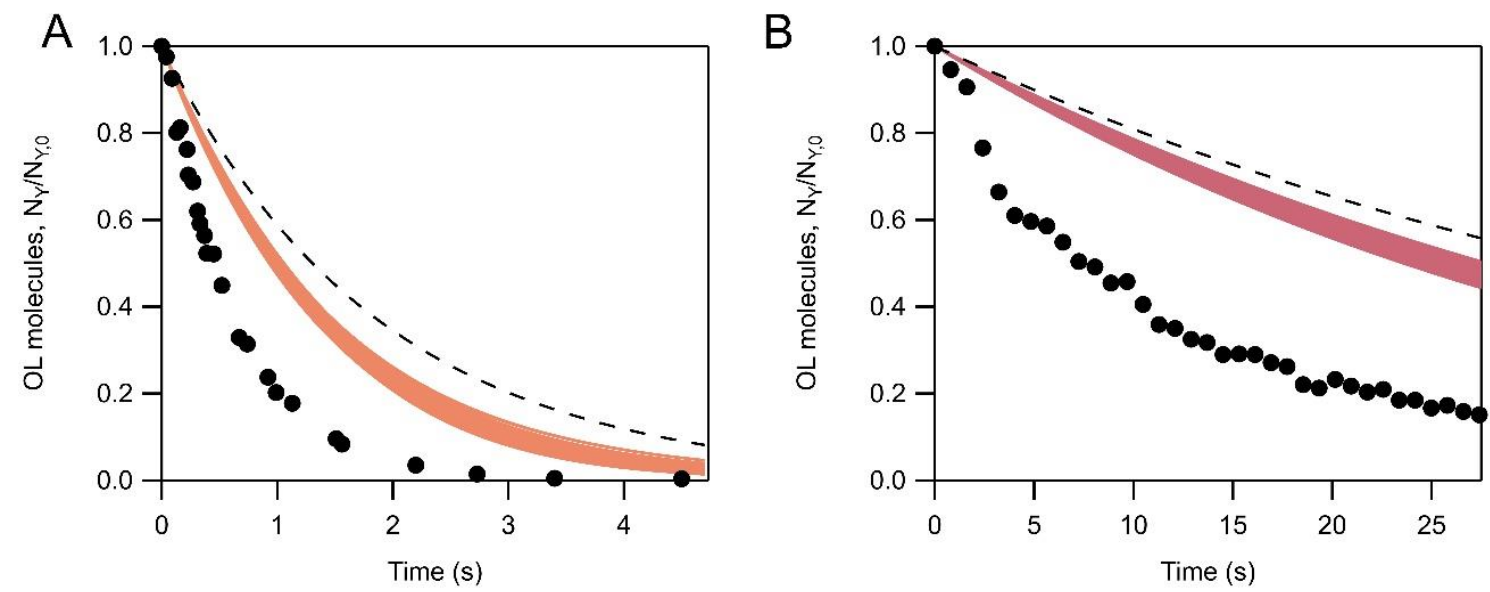

Figure S2: Model output according to modelling scenario A (dashed line) and modelling scenario B (ensemble of colored lines) for additional oleic acid ozonolysis data (black markers). (a) Data from Hearn et al. $2005^{1}$, which was recorded under similar experimental conditions as experimental data set FT1 (Hearn and Smith $2004^{2}$ ). (b) Data provided in exchange with P. Ziemann, including additional data not shown in the original publication (Ziemann 2005 ${ }^{3}$ ). 

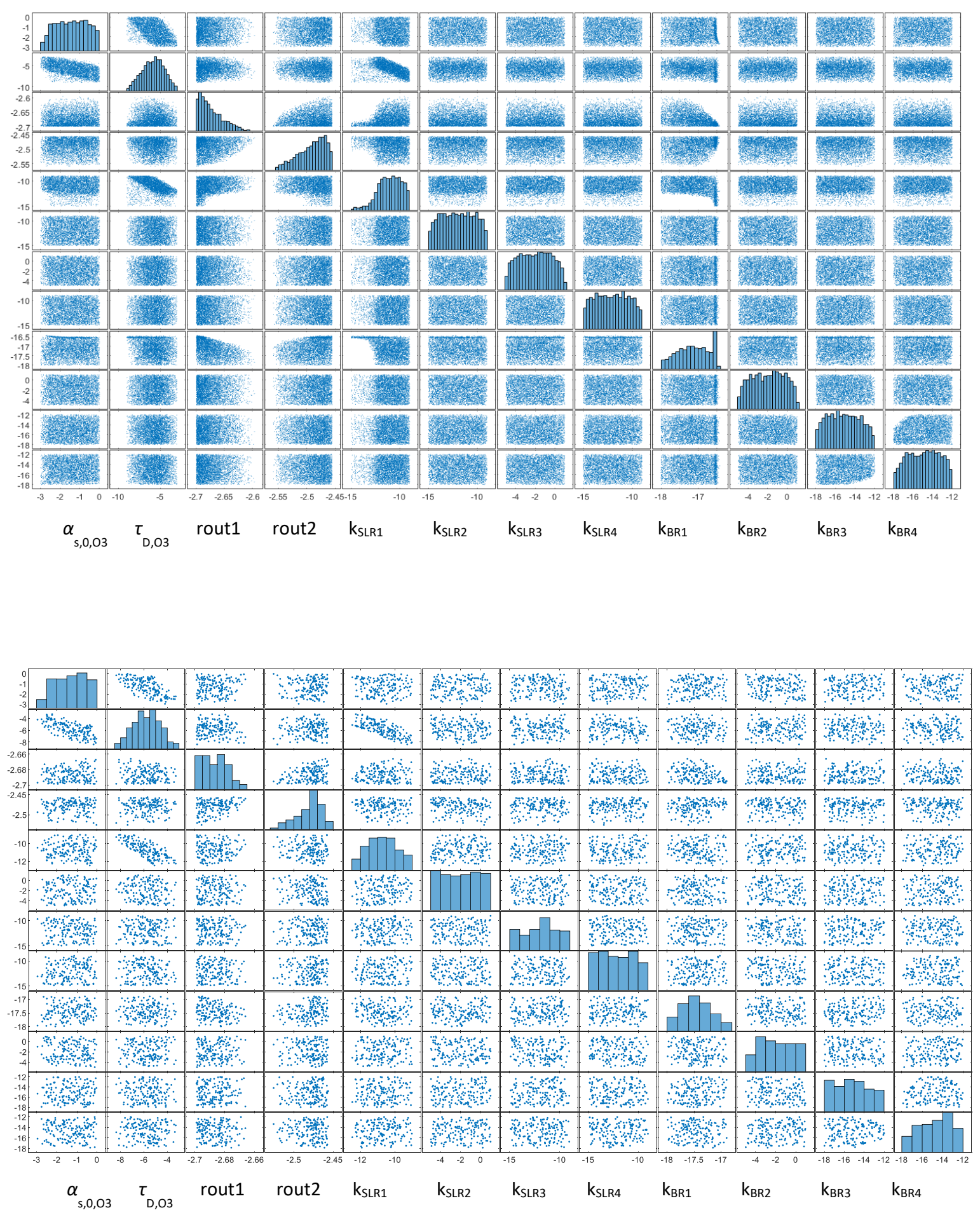

Figure S3: Scatter plot matrix of kinetic parameters of model fits according to modelling scenario $\mathrm{B}$ with (a) residual $\mathrm{R}<0.112(\mathrm{~N}=3995)$ and (b) residual $\mathrm{R}<0.108(\mathrm{~N}=167)$. Note that the boundaries of the individual plots do not correspond to the optimization boundaries but rather the minimal and maximal values returned upon optimization for each parameter. 


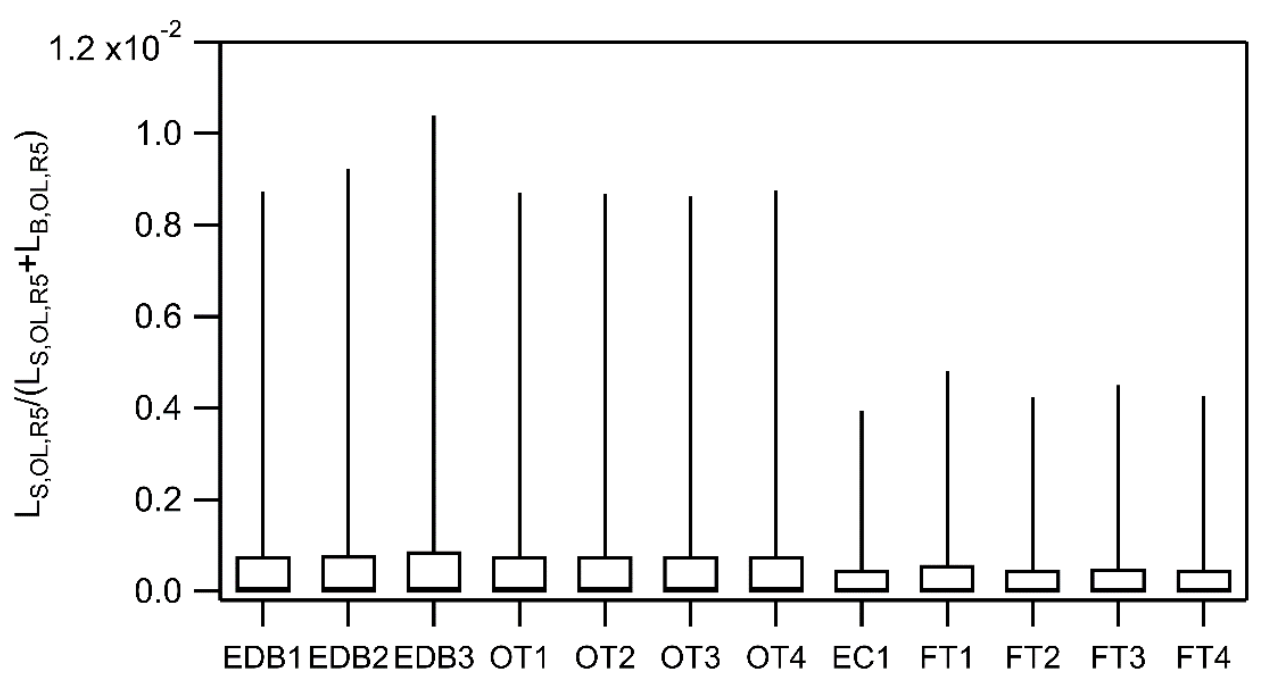

Figure S4: Surface-to-total OL loss ratio (STLR) for reaction R5 using KM-SUB model outputs for a host of $\mathrm{N}=167$ fits to a range of 12 experimental data sets using modelling scenario $\mathrm{B}$. The box-whisker plot shows the 10, 25, 50, 75 and 90 percentiles for each model output value.
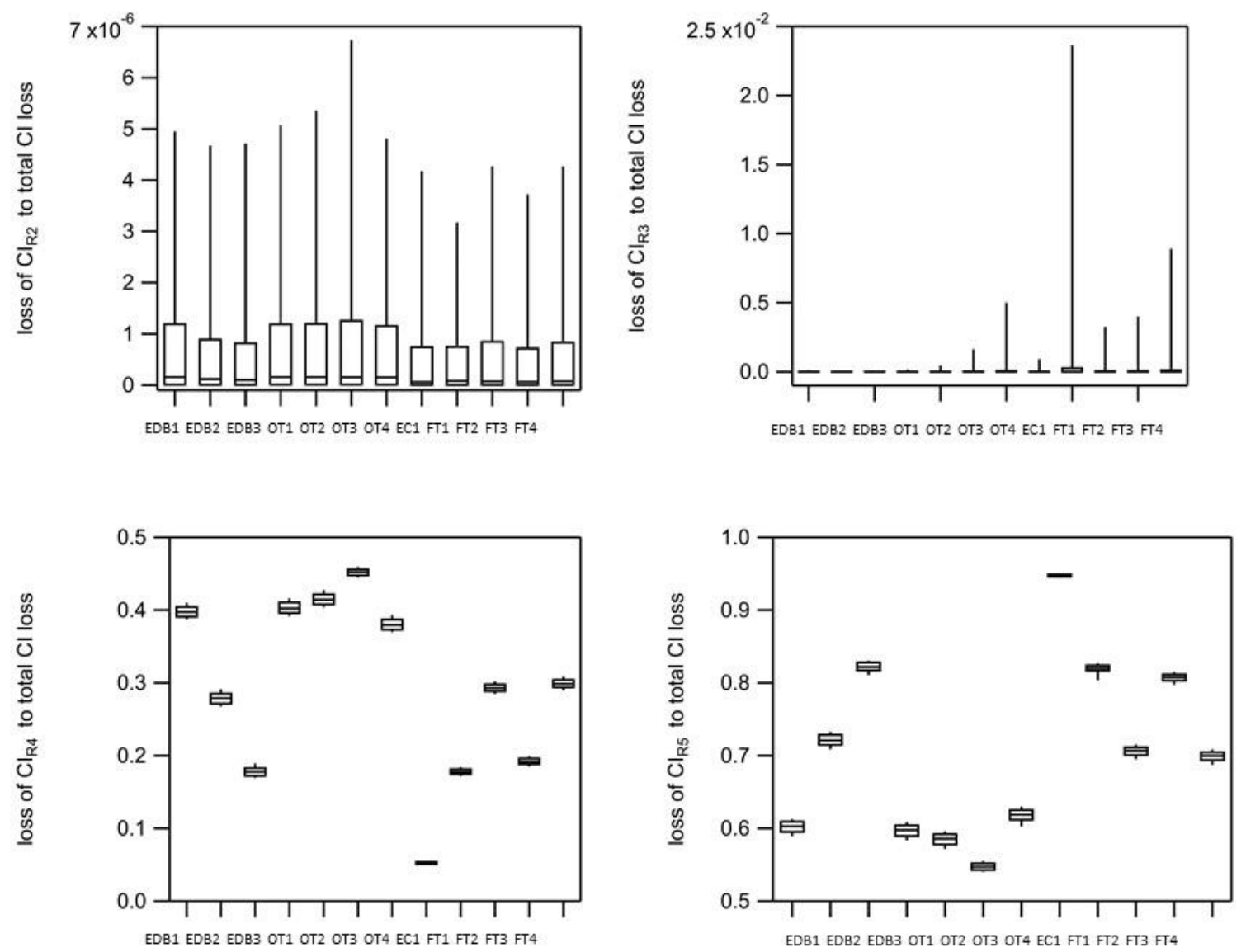

Figure S5: Relative CI fate for reactions (a) R2, (b) R3, (c) R4, and (d) R5 to total CI loss. The box-whisker plots show the 10, 25, 50, 75 and 90 percentiles for each model output value. 

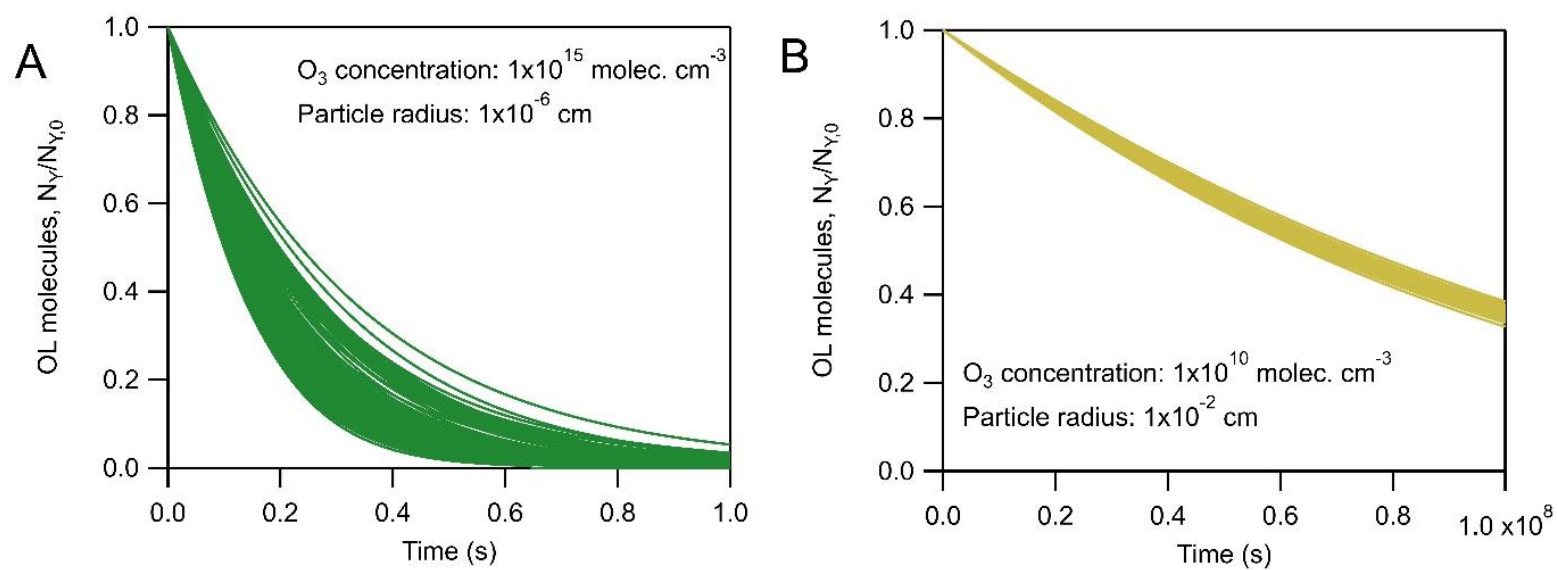

Figure S6: Model output for additional experimental conditions that have not been investigated experimentally. 
Table S1: Reaction mechanism used for modelling scenario $\mathrm{C}$, adapted from the template in Zeng and Wilson ${ }^{4}$.

$$
\begin{aligned}
& \mathrm{O} 3+\mathrm{OL} \rightarrow \mathrm{CI}+\frac{\mathbf{1}}{\mathbf{2}} \mathrm{C} 9-\mathrm{acid}+\frac{\mathbf{1}}{\mathbf{2}} \text { Nonanal } \quad \text { Fitted } \\
& \mathrm{CI} \rightarrow \mathrm{RO} \bullet+\mathrm{OH} \bullet \quad \text { Fitted } \\
& \mathrm{CI}+\mathrm{CI} \rightarrow \text { Peroxide } \quad \text { Fitted } \\
& \mathrm{CI}+\mathrm{C} 9 \text {-acid } \rightarrow \text { Peroxide } \quad \text { Fitted } \\
& \mathrm{CI}+\mathrm{OL} \rightarrow \mathrm{OL}^{*} \quad \text { Fitted } \\
& \mathrm{O} 3+\mathrm{OL}^{*} \rightarrow \mathrm{CI}+\frac{1}{2} \mathrm{C} 9 \text {-acid } \quad \text { Fitted } \\
& \mathrm{RO} \bullet \rightarrow \mathrm{C} 9 \text {-acid } \quad \text { Fitted } \\
& \mathrm{C} 9 \text {-acid }+\mathrm{OH} \bullet \rightarrow \mathrm{C} 9 \text {-acid } \quad 2 \times 10^{-11}, \operatorname{Ref}[1] \\
& \mathrm{OL}+\mathrm{OH} \bullet \rightarrow \mathrm{OL} \bullet \quad 2 \times 10^{-11}, \operatorname{Ref}[1] \\
& \mathrm{OL}+\mathrm{OH} \bullet \rightarrow \mathrm{CI}+\frac{1}{2} \mathrm{C} 9 \text {-acid } \quad 6.6 \times 10^{-11}, \operatorname{Ref}[1] \\
& \mathrm{OL}^{*}+\mathrm{OH} \bullet \rightarrow \mathrm{OL}^{*} \bullet \quad 2 \times 10^{-11}, \operatorname{Ref}[1] \\
& \mathrm{OL}^{*}+\mathrm{OH} \bullet \rightarrow \mathrm{CI}+\frac{1}{2} \mathrm{C} 9 \text {-acid } \quad 6.6 \times 10^{-11}, \operatorname{Ref}[1] \\
& \text { Peroxide }+\mathrm{OH} \rightarrow \text { Peroxide } \quad 2 \times 10^{-11} \text {, Ref [1] } \\
& \mathrm{C} 9 \text {-acid }+\mathrm{RO} \bullet \rightarrow \mathrm{C} 9 \text {-acid }+ \text { C9-acid } \quad 1.66 \times 10^{-15} \text {, Ref [1] } \\
& \mathrm{OL}^{*}+\mathrm{RO} \bullet \rightarrow \mathrm{C} 9 \text {-acid }+\mathrm{OL}^{*} \bullet \quad 1.66 \times 10^{-15}, \operatorname{Ref}[1] \\
& \mathrm{OL}+\mathrm{RO} \bullet \rightarrow \mathrm{C} 9 \text {-acid }+\mathrm{OL}^{*} \bullet \quad 1.66 \times 10^{-15}, \operatorname{Ref}[1] \\
& \text { Peroxide }+\mathrm{RO} \bullet \rightarrow \mathrm{C} 9 \text {-acid }+ \text { Peroxide } \quad 1.66 \times 10^{-15} \text {, Ref [1] } \\
& \mathrm{C} 9 \text {-acid } \bullet+\mathrm{C} 9 \text {-acid } \bullet \rightarrow \mathrm{C} 9 \text {-acid }+ \text { C9-acid } \quad 4 \times 10^{-15}, \operatorname{Ref}[1] \\
& \mathrm{C} 9 \text {-acid } \bullet+\mathrm{OL}^{*} \bullet \rightarrow \mathrm{C} 9 \text {-acid }+\mathrm{OL}^{*} \quad 4 \times 10^{-15} \text {, Ref [1] } \\
& \mathrm{OL}^{*} \bullet+\mathrm{OL}^{*} \bullet \rightarrow \mathrm{OL}^{*}+\mathrm{OL}^{*} \quad 4 \times 10^{-15}, \operatorname{Ref}[1] \\
& \text { C9-acid } \bullet+\text { Peroxide } \rightarrow \text { C9-acid + Peroxide } \quad 4 \times 10^{-15} \text {, Ref [1] } \\
& \text { Peroxide }+ \text { OL }^{*} \bullet \rightarrow \text { Peroxide }+ \text { OL }^{*} \quad 4 \times 10^{-15}, \text { Ref [1] } \\
& \text { Peroxide } \bullet+\text { Peroxide } \rightarrow \text { Peroxide }+ \text { Peroxide } \quad 4 \times 10^{-15}, \text { Ref [1] } \\
& \text { C9-acid } \bullet+\mathrm{C} 9 \text {-acid } \bullet \rightarrow \mathrm{RO} \bullet+\mathrm{RO} \bullet \quad 1 \times 10^{-16}, \mathrm{Ref}[1] \\
& \mathrm{C} 9 \text {-acid } \bullet+\mathrm{OL}^{*} \bullet \rightarrow \mathrm{RO} \bullet+\mathrm{RO} \bullet \quad 1 \times 10^{-16}, \operatorname{Ref}[1] \\
& \mathrm{OL}^{*} \bullet+\mathrm{OL}^{*} \bullet \rightarrow \mathrm{RO} \bullet+\mathrm{RO} \bullet \quad 1 \times 10^{-16}, \operatorname{Ref}[1] \\
& \mathrm{C} 9 \text {-acid } \bullet+\text { Peroxide } \rightarrow \mathrm{RO} \bullet+\mathrm{RO} \bullet \quad 1 \times 10^{-16}, \operatorname{Ref}[1] \\
& \mathrm{OL}^{*} \bullet+\text { Peroxide } \bullet \mathrm{RO} \bullet+\mathrm{RO} \bullet \quad 1 \times 10^{-16}, \operatorname{Ref}[1] \\
& \text { Peroxide } \bullet+\text { Peroxide } \rightarrow \mathrm{RO} \bullet+\mathrm{RO} \bullet \quad 1 \times 10^{-16} \text {, Ref [1] }
\end{aligned}
$$




\section{References}

(1) Hearn, J. D.; Lovett, A. J.; Smith, G. D. Ozonolysis of oleic acid particles: evidence for a surface reaction and secondary reactions involving Criegee intermediates. Phys.Chem. Chem. Phys. 2005,7, 501-11.

(2) Hearn, J. D.; Smith, G. D. Kinetics and Product Studies for Ozonolysis Reactions of Organic Particles Using Aerosol CIMS. J. Phys. Chem. A 2004,108, 10019-10029.

(3) Ziemann, P. J. Aerosol products, mechanisms, and kinetics of heterogeneous reac-tions of ozone with oleic acid in pure and mixed particles. Faraday Discuss. 2005,130, 469-90; discussion 491-517, 519-24.

(4) Zeng, M.; Wilson, K. R. Efficient Coupling of Reaction Pathways of Criegee Intermediates and Free Radicals in the Heterogeneous Ozonolysis of Alkenes. J. Phys. Chem. Lett. 2020, 11, 6580-6585. 